\title{
Das Register- und Konzeptswesen in der Reichskanzlei Maximilians I. bis 1502. \\ Von
}

Wilhelm Bauer.

Man hat die Zeit Maximilians I. bereits von den verschiedensten Gesichtspunkten aus mehr oder minder ausführlich behandelt, vom historisch-politischen, vom verfassungsgeschichtlichen und schliesslich vom literatur- und kunsthistorischen. Nur von Seite der Diplomatik, ist - ron einem einzigen Versuche abgehen ${ }^{1}$ ) - keine zusammenhängende Darstellung der Urkundenlehre für diese späte Epoche geliefert worden. Und dies hat seine guten Gründe. Fürs erste besitzt in dieser Zeit die Urkunde wirklich nicht mehr jenen quellenkritischen Wert wie einst und dann fehlen ja dem Bearbeiter so gut wie alle Vorarbeiten, nicht nur für die Kanzleigeschichte Maximilians selbst sondern auch für die seiner nächsten Vorgänger. Es bedarf also gewissermassen einer Rechtfertigung, wenn ich es versuche, einige rein diplomatische Fragen zu erörtern, die dem bereits ausgehenden Mittel-

1) Es ist dies der von S. Steinherz in den KU. i. A. (Text) S. 476 ff. gebotene kurze Abriss über das Urkundenwesen Maximilians I., der, abgesehen von äusseren Umständen nur das Wichtigste bieten und Vollständigkeit gar nicht anstreben konnte. - Überdies ligt im Archive des Instituts f. österr. Geschichtsforschung die Arbeit Dr. Ferd. Jančar's: Das Kanzleiwesen Maximilians I. als Manuskript verwahrt. Mein unvergesslicher Lehrer, Professor Mühlbacher, hatte die Güte, mir diesen Aufsatz für einige Zeit zu überlassen. Ich verdanke dieser im allgemeinen trefflichen Arbeit, über deren Vollendung der Verfasser frühzeitig gestorben ist, manche Anregung, doch verbreitet sie sich gerade über jene Themen, die mich interessirten, nur ganz vorübergehend. 
alter angehören. Die Bedeutung der spätmittelalterlichen Urkundenlehre liegt eben weniger darin, dass sie uns die Handhaben zur Bewertung des einzelnen Diplomes als geschichtliche Quelle gibt1), als vielmehr in der Fülle des Materials, das uns einen bedeutend tieferen Einblick in die Organisation und die Kanzleigebahrung gewährt als die notdürftigen Überreste der früheren Zeit. Dieser Einblick scheint mir nun, nicht nur wertvoll zu sein für die Erkenntnis, so weit sie sich auf die Gebräuche der betreffenden Kanzlei bezieht, sondern noch wichtiger, wenn sie uns Rückschlüsse auf vergangene Epochen gestattet $^{2}$ ).

Als Beleg für diese Ansicht darf man wohl auf die befruchtenden Anregungen hinweisen, die durch die Veröffentlichung der „ältesten Ordnung der deutschen Reichskanzlei“ ${ }^{3}$ ) auch für die Betrachtungen des Urkundenwesens vorhergehender Perioden einzelnen Forschern zu teil geworden sind. So findet sich Bresslau des öfteren veranlasst, die in dieser "Ordnung" festg. legten Normen der maximilianischen Reichskanzlei mit der Übung in früheren Kanzleien zum Vergleich heranzuziehen. Auch Seeligers verdienstrolle Arbeit über die "Registerführung am deutschen Königshofe bis $1493^{44}$ ) verdankt der Kenntnis der Kanzleiordnuugen von 1494 und 1498 manchen interessanten Hinweis auf Zustände und Verhältnisse, die lange vor der Zeit Maximilians bestanden haben.

Dies erfäbrt aber seine Begründung wie auch seine Berechtigung lediglich in der Tatsache, dass kaum ein Organ des öffentlichen Lebens so zäh an der Überlieferung festgehalten hat, wie eben die deutsche Reichskanzlei. Trotzdem wird man jene Bestimmungen von 1494 nicht kritiklos als Paradigma für die Gebräuche in früheren Kanzleien anwenden dürfen sondern nur mit einem gewissen Vorbehalt und stets mit genauer Rücksichtnahme auf die geänderten Verhältnisse.

Die Vorgeschichte dieser "Ordnung" ist bekanntlich folgende. Was seit Karl IV. ausser Übung blieb und Friedrich III. klug abzu-

1) Obwohl auch da noch die Frage nach der Echtheit der Urkunden eine grössere Rolle spielen kann, als man gemeiniglich anzunehmen geneigt ist. Vgl. Dvořak, Die Fälschungen des Reichskanzlers Kaspar Schlick in den Mitt. des Inst. 22. $51 \mathrm{ff}$.

2) In ähnlichem Sinne sprach sich bereits vor Seeliger Redlich aus geIegentlich der Rezension von Seeliger's, Das deutsche Hofmeisteramt im späteren Mittelalter in den Mitt. des Instituts 7, 494, wo er auch auf die Bedeutung hinweist, die eine Untersuchung der Reichsregisterbücher für die Auf bellung früherer Kanzleiverhältnisse hätte.

s) Archivalische Zeitschrift 13, 1 ff.

4) Mitt. des [nst. Erg.-Bd. 3. 
wenden wusste, musste Maximilian dem Mainzer Erzbischof zugestehen. Wohl oder übel musste er die alten Privilegien anerkennen und Bertold die Verwaltung der Reichskanzlei überlassen. Mit grösserer Entschiedenheit als 1440 der Trierer Erzbischof oder 1471-1475 Adolf von Mainz trat jetzt der streitbare Vorkämpfer für ständische Interessen an die Leitung der Kanzlei. Neue Kräfte zog er heran und eben daraus erklärt sich, warum er sich veranlasst sah, diese Ordnung zu erlassen. Bei der Kontinuität des Personals, wie es bisher bei einem Wechsel der Herrscher meist der Fall war, war es gewiss nicht nötig, die Grundsätze der Kanzleigebahrung so genau zu bestimmen wie diesmal. Es wäre aber ein Irrtum anzunehmen, Bertold habe in seiner Verordnung von 1494 Oktober 3 die Reichskanzlei neu organisiren wollen, vielmehr sprechen alle Anzeichen dafür, dass er darin nur die alte Tradition festgelegt habe. Wobl wurden mancherlei Bestimmungen aufgenommen, die bisher nur vortibergehend in Kraft waren, und Einrichtungen ins Leben gerufen, zu denen es früher nur schwache Ansätze gab, wie z. B. betreffs Tax- und Konzeptsregister. Doch darf man nicht rergessen, dass es sich hier um eine Kodifikation bergebrachter Zustände handelt, bei der, wie gewöhnlich, die schärfsten Seiten hervorgekehrt wurden. Es wird sich im Laufe dieser Erörterungen noch ergeben, wie man in der Reichskanzlei Bertolds selbst die Neuschöpfungen des Erzbischofs nur zögernd anfnahm und seiner Kanzleiordnung nicht in allen Stïcken mit der gewünschten Sorgfalt nachkam. Hiezu dauerte seine Amtsperiode vielleicht auch nur eine zu kurze Spanne Zeit. Iu den sechs Jahren bis 1500 konnten sich grössere Reformen eben nicht einleben und Bertold musste froh sein, wenn jene Punkte seines Programmes zur Ausführung gelangten, die auf die strenge Abhängigkeit der Kanzlei von ihm und auf die Wahrung ihrer Befugnisse gegenüber der Hofkanzlei hinzielten. Die Entfernung des wichtigsten Reichsamtes von den Sitze des Königs brachte bei den ohnehin gespannten Verhältnissen, die zwischen Maximilian und Bertold herrschten, stets neue Nahrung zu unerquicklichen Kompetenzstreitigkeiten. Den Höhepunkt erreichte dieser Kampf bekanntlich dureh Konstituirung des Nürnberger Reichsregimentes. Die Stände hatten den Bogen zu straff gespaunt. Am 21. März 1502 war der Laufbahn. Bertolds als Kanzler durch Abforderung des königlichen Siegels von Seite Maximilians jäh ein Ziel gesetzt ${ }^{1}$ ).

Alle Phasen dieses Streites lassen sich mittelbar auch in den Überresten dieser Kanzlei verfolgen und es ist oft nicht ohne einen

1) Über die Geschichte der Reichskanzlei vgl. G. Seeliger, Erzkanzler und Reichskanzleien (Innsbruck 1889) S. $62 \mathrm{ff}$. 
gewissen Reiz, die Reflexe der politischen Vorgänge in diesen scheinbar so abgelegenem Quellenmaterial zu beobachten.

\section{Das Registerwesen.}

Nirgends lässt sich vielleicht das Festhalten der Reichskanzlei an der Tradition so klar aufweisen als in der Geschichte der Registerführung. Nicht als ob nicht zu verschiedenen Zeiten auch verschiedene Besonderheiten zu Tage träten und dieser oder jener Kanzlei ihr eigenes Gepräge gäben, aber in den grossen Zügen bleiben sich doch alle spätmittelalterlichen Kanzleien hieriu sehr ähnlich. Dies lässt sich natürlich erst von jener Zeit an konstatiren, da wir das Material zienlich vollständig in einer fortlaufenden Reihe von Bänden erhalten haben, was ja erst von der Regierung König Ruprechts an der Fall ist. Wir werden nun sehen, dass die Registerführung der Reichskanzlei Maxinilians von der ererbten Überlieferung in keinem wesentlichen Punkte abwich. Es möge daher dieser Teil meiner Erörterungen nur als ein Anbang zu Seeligers Arbeit angesehen werden, zn dem sich noch einige Beobachtungen gesellen, die ich gelegentlich auch betreffs des übrigen Registermaterials gemacht habe ${ }^{1}$ ).

Die Anlegung von Registerbüchern, das sind, soweit es die Reichskanzlei betrifft, Bücher, die den Urkundenauslauf verzeichnen, erfolgte gewiss aus rein praktischen Gesichtspunkten. Aber die Schwerfälligkeit, die der Verwaltungstechnik damals noch auf allen Gebieten eigen war, zeigt sich auch hier klar und deutlich. Gerade dem Haupterfordernisse, der Vollständigkeit und Übersichtlichkeit, hatte die Reichsregistratur nur in geringem Masse Rechnung getragen. Und doch hatte in Bezug auf praktische Anordnung die Kanzlei Ruprechts, der die ersten Bände unserer Registerreihe angehöreu ${ }^{2}$ ), mehr geleistet als die folgenden. Man schied die Bücher sowohl nach der Sprache, in welcher die verzeichneten Urkunden abgefasst waren, als auch nach deren Besiegelung ${ }^{3}$ ). Später empfand man ab und zu auch das Bedürfnis nach einer Abgrenzung gleichartiger Urkundengruppen, indem man ihnen eigene Bände zuwies. Dies geschah nun freilich in der Kanzlei Ruprechts noch nicht, doch werden wir an anderer Stelle nachweisen können, dass sich wenigstens Ansätze hiezu deutlich verfolgen lassen.

1) Bei Bearbeitung der XII. und XIII. Lieferung ven Chrousts Monumenta palaeographica, bei der ich bestrebt war, in den ausgesuchten Tafeln, sowohl vom paläographischen wie diplomatischen Standpunkt den Lauf der Entwicklung in der Registerfuhrung zu reranschaulichen.

2) Wobei wir von den Fragmenten aus früherer Zeit absehen.

9) Seeliger, Registerführung S. 353. 
1. Die Registerbüicher. Nur der Vollständigkeit halber seien zuuächst zwei Bände kurz beschrieben, die der Zeit angehören, bevor Bertold die Leitung der Reichskanzlei übernommen hat. Da sie Seeliger in seine Arbeit nicht einbezog und sie sonst kaum beachtet werden dürften, solleu sie im nachfolgenden mit wenigen Worten gestreift werden. Das ältere dieser zwei Registerbücher ist Band EE. Es ist dies ein unpaginirter Pergamentkodex (im Formate $265 \times 370 \mathrm{~mm}$ ), der in Quinternionen gelegt erscheint und inhaltlich in zwei selbständige, wenn auch verwandte Teile zerfällt, die wir kurz I und II bezeichnen wollen.

$\mathrm{EE}$ ist uun ein Aktregister, das lediglich zur Aufnahme Erster Bitten bestimmt war, welche alphabetisch nach dem Ortsnamen der Kirchen oder dem Namen des Kirchenheiligen angeordnet waren. Die Vorsteckblätter sowohl vor I wie vor II weisen die gleiche Aufschrift auf, nämlich: Registrum primariarum precum, que emanaverunt a Romanorum rege domino Maximiliano Austrie archiduce, Burgundie duce etc. de anno domini millesimo quadringentesimo octuagesimo sexto. Auch sonst stimmen inhaltlich beide Teile im grossen und ganzen überein. Bei näherer Prüfung freilich ergibt sich, dass in Wirklichkeit nur II in Jahre 1486 angelegt worden ist $u$, zw. von einem "meister Arnolt" "). Das Jahr der Anlage ergibt sich nämlich aus dem Umstand, dass durch das ganze Heft hindurch die Akte aus diesem Jahre von einer Hand gleichmässig eingetragen erscheinen, von da an sich aber Nachträge bis 1488 konstatiren lassen.

Teil I ist trotz der Überschrift auf dem Vorsteckblatte, die gedankenlos von II herübergenommen wurde, im Jahre 1491 angelegt und bis 1495 mit Nachträgen versehen. Den Grundstock von I bildet jedoch die Abschrift von II, wobei die in I ausgestrichenen oder mit „dimisit" bezeichneten Akte ausgelassen, dagegen vielfach neue hinzugefügt wurden.

Es bilden diese zwei Registerbände ${ }^{2}$ ) in der ganzen Reihe der Reichsregister durch ihre Anordnung eigentlich ein Unikum.

Weniger Beachtung als EE gebührt dem Bd. FF, der aus 143 Papierblättern im Formate $215 \times 305 \mathrm{~mm}$ besteht. Es ist dies ein allgemeines Register, dessen Eintragungen zum grössten Teil aus den Jahren 1490 und 1491 herrührt.

1) Dies geht aus Notizen auf dem Schlussblatte von II hervor. Dort werden nämlich Nachträge zu dem -Register gebracht, unter denen es einmal heisst: die all sten in moister Arnolt register nicht.

2) Als solche sind: I und II, obwohl sie jetzt unter einer Signatur erscheinen, doch zu betrachten. 
Unstreitig grössere Berücksichtigung verdienen die zwei Pergamentbände, die hente die Signatur $\mathbf{X} 1$ und $\mathbf{X} 2^{1}$ ) tragen, die aber noch zur Zeit Maria Theresias ein einziges grosses in Schweinsleder gebundenes Buch bildeten, das bloss mit X bezeichnet wurde 2 ). Daraus ist es eben auch erklärlich, dass beide Bände das gleiche Format $(290 \times 370 \mathrm{~mm})$ besitzen und fortlaufend foliirt sind. X 1 besteht aus $376, \mathrm{X} 2$ aus 352 Blättern. Dieselben sind bis $\mathrm{Bl}$. $40^{\prime}$ in verschiedenen Lagen geordnet, von da an folgen in X 121 0kternionen, während X 2 aus 22 solchen besteht. Neben der ungefähr gleichzeitigen Folienzählung finden sich auch Spuren einer ursprünglichen Lagenbezeichnung. Schon in $\mathrm{X} 1$ sind die ersten vier Lagen mit Buchstaben des Alphabets versehen ${ }^{3}$ ). Dann trifft man wieder auf Bl. 121 links unten die Zahl 2, am Anfangsblatt des nächsten Okternio (Bl. 137) die Zahl 3 und Bl. 153 wieder 4. Damit verlieren sich für $\mathrm{X} 1$ die Spuren einer Lagenzählung. Eine solche beginnt erst wieder in $\mathrm{X} 2$ mit dex zeinnten Lage Bl. 527, wo links unten die Zahl 36 . angebracht erscheint. Diese Zählung läuft dann in den folgenden 7 Okternionen weiter ${ }^{4}$ ). Die Zahlen sind oft nur zu erraten, da der Kodex beim Binden beschnitten wurde, womit auch ihr sporadisches Auftreten sich erklärt. Immerhin rätselhaft bleibt es, von welchem Anfangspunkte aus gerechnet wurde, denn beginnt man mit X 1 die Lagen fortlaufend zu zählen, so kommt man bei Bl. 527 nicht zur 36. sondern zur 34. Lage.

Sachlich teilt sich der Bd. $\mathrm{X}$ in 6 bezw. 7 streng von einander geschiedene Abteilungen. Die erste hievon umfasst deu ganzen $B d . X_{1}$ und reicht in $\mathrm{X} 2$ bis Bl. 458, wo auch die 10. Lage dieses Kodex endet. Sie enthält ein s. g. allgemeines Register vorzugsweise Privilegien und Bestätigungen. Daran schliessen sich 11 Lagen, in denen durchwegs Lehenbriefe registrirt wurden. Diese Abteilung reicht bis Bl. 636. Dass die letzten zwei Blätter der 11. Lage (Bl. 637 und 638). unbeschrieben blieben, bekundet um so deutlicher die Selbständigkeit dieses Lehenregisters innerhalb des ganzen Buches. Mit Bl. 639 hebt ein neuer Abschnitt an, der sich bereits durch die Überschrift „Quittanzen" als solcher einleitet. Ihm ist eine Lage gewidmet von der die 4 letzten Blätter leer blieben. Der nächste Okternio stellt sich wieder als ein für sich abgeschlossenes Heftchen dar, das mit folgender

1) Vgl. hiezu meine Beschreibung in Chrousts Monumenta palaeogr. XII, 10. Der Vollständigkeit halber waren Wiederholungen unerlässlich.

2) Wien, H. H. St.-A. Verwaltungsakten: Rosenthals Bericht 1751.

3) Bl. 1' mit a, Bl. 13' mit c, Bl. 17' mit b, Bl. 29' mit d.

4) X 2 Bl. 543, 559, 575, 591, 507, 623, 639 . 
Überschrift einbegleitet wird: Hierinne sein registrirt die hanndlungen und verschreibungen zwischen unnsern allergnedigisten herrn dem Romischen kunig etc. und hertzog Albrechten von Sachssen etc. der Friesischen lannde halben ". Von Bl. 671 bis 708 folgen verschiedene Privilegien, meist Jahrmarkts- und Zollverleihungen, Bl. 708-714 blieben unbeschrieben, die Blätter 715-723 enthalten Privilegien für Württemberg. Von Bl. 724-727 folgt der Index, der wenig später als die beiden Kodizes angelegt worden ist.

So verschiədenen Inhalts diese Abteilungen auch sein mögen, ihre Anlage fällt - ausgenommen die Verträge mit Sachsen - ungefähr in die gleiche Zeit. Diese Register wurden nämlich nicht streng gleichzeitig mit der Ausfertigung der eingetragenen Urkunden geführt und auch nicht gleich mit Beginn der Kanzleiführung des Mainzers. angefangen. Fassen wir zunächst das allgemeine, das Lehens- und Quittungsregister ins Auge, so bemerken wir bei allen dreien stets zu Anfang eine krasse Unordnung in der zeitlichen Reihenfolge; dies ändert sich später, doch schieben sich bis zum Schlusse Nachträge aus den frühesten Zeiten ein, wobei trotzdem das eine oder andere Jahr vorherrschend bleibt. Verfolgt man dies nun beim allgemeinen ${ }^{1}$ ), beim Lehen ${ }^{2}$ ) und Quittungsregister ${ }^{3}$ ), so fällt es auf, dass die erwähnten chronologischen Unregelmässigkeiten mit dem Jahre 1496 aufhören und von da an wohl Nachträge aus früheren, nicht aber Einschübe aus späteren Jahren vorkommen. Daraus ergibt sich mit einiger Bestimmtheit, dass die Anlage frühestens 1496 stattgefunden haben mag. Der letzte Teil, der vorzugsweise Jahrmarktsprivilegien enthält, scheint Ende 1495 angelegt worden zu sein.

Gemäss der Vorschrift der Kanzleiordnung4) sollten die Regesten mit den Konzepten verglichen werden. Tatsächlich treffen wir nun an verschiedenen Stellen des Registers Vermerke wie ${ }_{n}$ coll..45) oder "coll. per totum ${ }^{6}$ ), die mit collationatum aufzulösen sind. Von wenigen Ausnahmen abgesehen finden sich diese Kollationsvermerke stets auf dem ersten Blatte der einzelnen Lage, was wohl darauf hindeutet, dass die Überprüfung lagenweise vorgenommen worden ist.

Schliesslich sei noch erwähnt, dass sich in der Reihe der Reichsregister zwei Papierbände vorfinden, die mit der Aufschrift „Kaiser

1) Dieses umfasst die Jahre 1493-1501 Oktober.

2) Hier reichen die Eintragungen von 1494 Juni 18 bis 1501 September 6 . Februar.

3) Die Eintragungen bewegen sich da zwischen 1494 Juli 4 und 1502

4) Seeliger II, 2.

5) $\mathrm{Bl} .41,57,73,89,105$ etc.

6) Bl. 153, 169, 184', 185 etc. 
Maximilian I. Reichsregistratur. Liber I“ bezw. „Liber II" versehen sind, Diese decken sich inhaltlich vollständig mit X 1 und $X 2$, kennzeichnen sich aber als spätere Abschriften durch die Tatsache, dass die in $\mathrm{X}$ vorkommenden Kanzleivermerke hier nicht von einer zweiten Persönlichkeit sondern vom Schreiber selbst eingetragen und mauche Fehler in $\mathrm{X}$ hier stillschweigend richtig gestellt wurden.

2. Zeitpunkt und Form der Eintragungen. Die Kanzleiordnung von 1494 bestimmt ausdrücklich, dass der Registrator alle unter anhangendem Siegel ausgehenden Stücke in ein Buch eigenhändig eintragen oder doch auf seine Kosten von anderen verzeichnen lassen, und die Eintragungen mit den Konzepten kollationiren soll. Er muss ferner auf alle Urkunden und besonders auf die mit anhangendem Siegel „aussen zuruck“ (also auf der Rückseite) das Wort „Registrata“ und seinen Namen schreiben. Daraus ginge es noch nicht klar hervor, welche die Vorlagen waren, die der Registrator zugrunde legte, hiesse es nicht des weiteren in den Vorschriften für die Sekretäre, sie sollen die „concept oder minuten", welche registrirt werden müssen, „dem registrator behenndigen ".

Die Durchforschung der Registerbücher stellt ausser Zweifel, dass. die aus früherer Zeit gewonnenen Ergebnisse auch für die Reichskanzlei Maximilians volle Geltung haben, dass nämlich zumeist Konzepte als Vorlage für die Eintragungen gedient haben.

Als Beweis hiefür dient einmal das oftmalige Vorkonmen von Regesten ohne die Datirungsformel ${ }^{1}$ ), was wohl deutlich darauf hinweist, dass die Abschrift nicht nach dem Orignal vorgenommen worden ist. Aus späteren Erörterungen wird man ersehen, dass es Regel war, dem Konzept die Datirung erst im letzten Stadium anzufügen uud es nicht selten vorkam, dass man davon überbaupt absab.

Aber noch deutlicher finden wir die Frage nach der Vorlage beantwortet, wenn bei solchen undatirten Stücken der Registrator vermerkt: „datum non reperitur in copia“" ${ }^{2}$ ), wobei darauf hingewiesen sei, dass damals das Wort copia ganz gewöhnlich für Konzept gebraucht wunde. Ja, oft weisen diese Bemerkungen direkt auf die Person des Konzipisten der deutschen Stücke hin, wenn fast stereotyp wiederkehrt "datum non invenitur (reperitur) in copia Johannis Storehen*3), oder ,ita habetur in copia absque dato Storch*4).

1) Z. B. X 1 Bl. $76,76^{\prime} 77^{\prime}, 78^{\prime}, 82^{\prime}, 88$ u. v. a.

2) $\mathrm{X} 2 \mathrm{Bl} .503,548,636$.

s) X 2 Bl. 499', 500' 503; X I Bl. 144:, lst kein datum bey Storchen coppey funden .

4) $\mathrm{X} 1 \mathrm{Bl}, 143$ 
Auch Notizen wie, ,acat und stet die recht eopey hievor registriert 1) u. a. sind deutliche Fingerzeige dafür, dass es in diesen Fällen nicht die Originale waren, die dem Registrator bei seiner Arbeit vorgelegen sind. Dass dies fast Regel war, beweist die zahlreiche durch beide Bände zerstreute Wiederkehr solcher Anmerkungen, wodurch der Registrator oder vielleicht der Kollationator - denn oft rähren sie von anderer Hand her als der Text der Regesten ${ }^{2}$ ) - sich zu rechtfertigen sucht und die Schuld der Mangelhaftigkeit auf den Konzipisten schiebt.

Aber abgesehen von diesen Vermerken ergibt sich aus dem, was vorhin über die Anlage der Register gesagt.wurde, der stringente Beweis, dass ein grosser Teil der daselbst verzeichneten Stäcke nach dem Original gar nicht eingetragen werden konnte, weil diese Eintragungen ja erst einige Jahre später stattgefunden haben. Wie oben angegeben wurde, umfasst X Stücke von 1493/4-1501/2. Die Anlage des Registerbuches erfolgte aber 'frühestens 1496. Es lagen also zwischen der Ausfertigung der ältesten Urkunden und ihrer Registrirung nicht weniger als zwei bis drei Jahre.

Immerhin mag ab und za auch nach dem Original registrirt oder dieses doch zur Kontrolle herangezogen worden sein, wenn wir nämlich Kanzleiunterfertigungen verzeichnet finden ${ }^{3}$ ), wie: „ad mandatum domini regis in consilo - Bertholdus archiepiscopus Moguntinensis, archicancellarius per Germaniam subscriptus“4) oder „Ad mandatum domini regis proprium - Bertholdus etc. ${ }^{5}$ ).

Die Form der Regesten weicht nicht wesentlich von der in früheren Zeiten beobachteten ab. In der Regel beginnen die Urkunden, wenn sie deutsch sind, mit "Wir Maximilian etc." Nur, wenn es sich um eine feierliche Ausfertigung handelt, wird noch angemerkt "cum“ oder "sub maiori titulo" ${ }^{*}$ ) oder ,cum pleno titulo" 7 ) einmal aber auch ,cum minori titulo ${ }^{8}$ ).

Ausführlich trägt man bloss die Disposition ein und selbst bei dieser weisen die verstreut vorkommenden "etc." darauf hin, dass man alles, was als formelhaft dem Kanzleischreiber geläufig war, wegliess. Dies hängt wohl auch mit der Fassung der Konzepte zusammen.

1) $\mathrm{X} 2 \mathrm{Bl} .534^{\prime}$.

2) Z. B. X $2 \mathrm{Bl} .499^{\prime}, 503,534^{\prime}$ etc.

3) Vgl. Seeliger, Die Registerführung $\mathrm{S} .330 \mathrm{ff}$.

4) X 1 B], 20', 23,43',81'.

$\left.{ }^{5}\right) \mathrm{X} 1 \mathrm{Bl} .38,38^{\prime}, 39,39^{\prime}$ etc.

6) Z. B. X 1 Bl. 5, 51, 54, 68' $81^{\prime} 86$ etc.

ๆ) $\mathrm{X} 1 \mathrm{Bl} .8$.

8) X 1 Bl. 189. 
In vielen Fällen begnügte man sich überhaupt nur mit einem Auszug besonders bei Urkunden, deren Formular ein feststehendes war, wie bei Legitimationen, Schirmbriefen, Belehnungen, Jahrmarktsprivilegien und Dienstbriefen. Zur Regel wurde diese auszugsweise Wiedergabe bei sogenannten "Generalconfirmacien", wenn diese nicht in besonders feierlicher Form ausgestellt wurden. Wenn zwei oder mehrere Stücke aufeinanderfolgen, deren Text sich nur in den individuellen Angaben unterscheiden, so wird bei dem zweiten Regest einfach mit „In simili . ." auf das erste, vollständiger mitgeteilte, Bezug genommen.

Wie man sieht, wurde von der Tradition, die in den vorhergehenden Kanzleien herrschte, in keiner Weise abgewichen.

3. Die Registerbücherals Hilfsmittel des Verwaltung. Während Maximilian in seinen Erbländern von Reform zu Reform eilte und den alten Verwaltnngsmechanismus mit neuem Leben erfüllte, blieb die Reichskanzlei - solauge wenigstens Erzbischof Berthold ihr vorstand - von dem neuen Geiste unberührt. Was Seeliger an der Registerführung bis 1493 in Bezug auf ihre administrative Verwendbarkeit auszusetzen hatte, gilt in vollem Masse auch für die folgende Zeit. Ansätze zu Verbesserungen sind deutlich in der Kanzleiordnung von $1494 \mathrm{zu}$ verfolgen. Danach sollten Bücher für Briefe über Provisionen, Diener und Amtleute geführt (IV., 9), ein eigenes Register für Tagsatzungen, Landeshuldigungen und Passbriefe (IV., 10) und ein solches für die Tuxen angelegt werden (V., 5); aber nichts ron alledem ist uns erhalten und es bleibt fraglich, ob man überhaupt daran ging, diese Vorschriften zu verwirklichen. Auch in anderen Punkten wich man ja von der Befolgung jener ab. Wären uns $X 1$ und $X 2$ verloren gegangen, wir müssten uns den Gang der Registerführung in manchen Punkten wesentlich anders vorstellen. In der Kanzleiordnung von 1494 heisst es ausdrücklich, die Eintragungen hätten in ein Buch zu erfolgen. Danach müsste man nun folgerichtig den Schluss ziehen, man habe in der Reichskanzlei von da an wirklich in festgebundene Bücher registrirt ${ }^{1}$ ).

Wie stand es nun damit in früherer Zeit? Seeliger S. 341 erklärt: "Noch ein Anderes ist aber aus den letzten Jahrzehnten Friedrichs als merkwürdig hervorzuheben. Die Register CC und DD zeigen, dass damals mitunter die Registratoren - nicht umfangreiche Codices, sondern kleine Heftchen für ihre Aufzeichnungen verwandten.

1) Dies folgert auch Neudegger, System und Systematisierung der Papst-, Kaiser- und Landregister (S. A. aus Bayer. Archivrepertorien und Urkundenregister, München 1900) S. 193 und er fügt hinzu: , Aus dieser unscheinbaren Bestimmung resultirte eine Umwälınng im Registerwesen c. 
Und das hat gelegentlich schon viel früher stattgefunden". Er weist dann an den Ruprechtsregistern 520, 538, 548 und 592, ferner an den Bänden $\mathrm{C}, \mathrm{E}$ und $\mathrm{N}$ nach, dass auch diese nicht in fertige Bücher geschrieben worden sein dürften. Auch Lechner machte bei der Beschreibung des von ihm aufgefundenen landesherrlichen Registerbuches Friedrichs III. eine ähnliche Beobachtung ${ }^{1}$ ), präzisirte aber die Bestandteile des Bandes bereits genaner als "Lagen".

Ich selbst habe nun gelegentlich der Durchsicht verschiedener Registerbände aus früherer Zeit ${ }^{3}$ ) die Erfahrung gemacht, dass auch noch in manchen anderen Kodices deutliche Spuren sich verfolgen lassen, die die Herkunft der Bände aus zusammengehefteten Lag en beweisen. So schliesst in Band A die Abteilurg der untcr Majestätssiegel ausgehenden Urkunden gewiss nicht zufällig gerade mit der 10. Lage $\mathbf{a b}^{3}$ ) und auch die Absonderung der acht Lagen des Bandes $G^{4}$ ) durch Einschiebung von Pergamentblättern deutet auf die Entstehungsweise dieser Kodices hin. Desgleichen konstatiren wir bei $\mathrm{H}$ (wie auch bei $E$ und $G$ ) eine deutliche Lagenzählung. Auch wird man es keinem blossen Zufall beimessen dürfen, wenn die nach den verschiedenen Kanzlerschaften abgesonderten Teile von 0 auch in ihrer Lagenanordnung scharf getrennt sind. Dies dürften die markantesten Fälle sein, an denen es sich nachweisen lässt, dass die betreffenden Bände in der Weise entstanden sind, dass man Lage anf Lage beschrieb und diese erst später zu den heute vorliegenden Büchern zusammenfasste ${ }^{5}$ ).

Vergleichen wir nun die Beobachtungen über die Registerführung der vorangehenden Kanzleien mit dem Gebrauche, wie er unter Erzbischof Bertold üblich war, so ergibt sich, dass die Reichskanzlei mit Übergehung der Anordnung von 1494 der alten Tradition foigte. Die Beschreibung von $\mathrm{X}$ hat zur Genüge ergeben, dass dieser Kodex aus der Vereinigung einzelner Lagen entstanden ist.. Für den praktischen Gebrauch der Register als Verwaltungsbücher brachte die lagenweise Anordnung manche Nachteile, von denen nicht der geringste die stete Gefahr war, die losen Lagen könnten in Verlust geraten. Dass dies auch wirklich geschah, zeigen die heute als CC bezeichneten zwei

1) Mittheilungen des Instit. 20, 55 .

2) Bei. Bearbeitung der XII. Lieferung der Monumenta palaeographica herausgeg. von A. Chroust.

y) Vgl. Mon. palaeogr. XII, 1.

4) Ebda XlI, 5.

5) Bd. S, der in der Zeit, da der Erzbischof Adolf von Mainz die Reichskanzlei verwaltete, entstand, ist tatsächlich als ein , Buch * zu bezeichnen, in das man die Eintragungen machte. Also geradezu eine Ausnahme. 
Sexternionen, die beide Bestandteile vielleicht verlorengegangener Registerbücher darstellen.

Anderseits hatte die Eintragung nach Lagen gewisse Vorteile, besonders dort, wo man an eine Gliederung nach Urkundenarten dachte. Da konnte man gleichzeitig damit beginnen, die verschiedensten Stücke in der betreffenden Abteilung einzutragen und hatte schliesslich den ganzen Regestenvorrat eines bestimmten Zeitraumes in einem Bande beisammen. Für die administrative Verwertbarkeit wäre es freilich besser gewesen, die verschiedenen Abteilungen (wo man solche überhaupt berïcksichtigte) zu sammeln und aus mehreren Jahren die Lagen der Lehens-, Quittungs- oder ähnlicher Urkunden zusammenzufassen ${ }^{1}$ ).

Im übrigen waren die Registerbücher auch in anderer Hinsicht nicht ganz zweckentsprechend. Besonders an der getreuen Wiedergabe der Stücke mangelte es vielfach. Liegt schon in der Neuerung, dass die Urkunden nicht nach dem Itinerar des Königs sondern nach dem jeweiligen Aufenthaltsort der Kanzlei datirt worden sind ${ }^{2}$ ) Anlass genug zu Irrtümern, so war es noch verhänguisvoller, wenn aus den früher erwähnten Gründen vielfach das Datum vollständig fehlte. Aber selbst wo die Vorlage datirt war, unterliess manchmal der Registrator die Angabe der gemeinen Jahre ${ }^{3}$ ). Trotz der Kollation liess man eben noch genug Verstösse unkorrigirt.

Nur $a b$ und zu verrät die Bemerkung einer fremden Hand wie: que est penitus falsa ${ }^{4}$ ), dass man das Registerbuch hie und da zu Vergleichen heranzog. Mag es in diesen Fällen noch zweifelhaft sein, ob dies nicht der Kollationator vermerkt hatte, so zeigt das Lehenregister in $\mathrm{X} 2 \mathrm{ganz}$ deutlich, dass man da die Regesten in steter Evidenz hielt, um Veränderungen nachzutragen. So finden wir Bemerkungen wie "hat glubd getan und ist der cantzley zugeschriben“ " ) oder "ist zugeschriben das er lehenpflicht getan hat" ${ }^{6}$ ) oder ähnliche Notizen, die erst später beigefügt wurden und von der praktischen Verwendung wenigstens dieses Teiles der Register deutlich Zeugnis ablegen ${ }^{2}$ ).

1) Nur den preces primariae widmete man, wie wir gesehen haben einen eigenen streng abgesonderten Band (EE), doch finden sich deren auch in $\mathbf{X}$.

2) Vgl. Stälin, Forschungen z. d. Geschichte 1, 349 und V. v. Kraus, Archiv f. österr. Gesch. 87, $231 \mathrm{ff}$.

s) Z. B. X BI, 18 .

4) Z. B. X Bl. $140^{\prime}$.

5) X 2 Bl. 504, 521, 547, etc.

6) $\mathrm{X} 2 \mathrm{~B}$. $\check{5} 05$.

¡) Vgl. Woldemar Lippert, Die deutschen Lehnbücher (Leipzig 1903) S. $100 \mathrm{ff.}$ 


\section{Das Konzeptswesen.}

Konnten sich die Erörteruugen über das Registerwesen nur in den Grenzen einer Fortsetzung bezw. Ergänzung zu der inhaltsreichen Arbeit Seeligers bewegen, so bietet das Material, das für die nachfolgenden Darlegungen verwertet wurde, ungleich mehr Neuartiges als die Register. Wie die Überschrift besagt, soll hier die Konzeptsgebahrung in der Reichskanzlui Maximilians behandelt werden.

Dieser Teil der Urkuudenlehre wurde scheinbar manchmal übergangen. Bei näherem Zusehen erkennen wir freilich, dass für frühere Zeitläufte so gat wie jedes Material für die Behandlung dieser Frage fehlt. Von einigen ganz vereinzelten Fällen abgesehen sind uns erst seit Heinrich VII. wirkliche Kanzleikonzepte für Königsurkunden überliefert ${ }^{1}$ ). Doch auch aus den folgenden Kanzleien sind uns nicht festgefügte Bestände dieses Quellenmaterials erbalten, sondern meist spärliche Reste, die wir nur dem Zufalle verdanken. In den Registerbïchern Sigismunds, Albrechts und Friedrichs III. findet sich wohl eine Anzahl solcher Stücke eingeklebt ${ }^{2}$, doch ist sie zu gering, um uns über das Konzeptswesen dieser Zeit sichere Aufschlüsse zu geben. Erst aus der Epoche Friedrichs III. häuft sich die Menge der erhaltenen Konzepte, von denen auch ein Teil Reichssachen betrifft. Es liegen solche sowohl in k. u. k. Staatsarchiv in Wien, wie auch im k. k. Statthaltereiarchiv in Innsbruck.

Eine mehr oder minder geordnete Sammlung von Urkundenentwürfen, die der Reichskanzlei entstammen, findet sich aber zum ersten Male aus der Zeit Maximilians vor. Es sind dies die in der Serie der Reichsregistratur eingereihten Konzeptsbücher.

1. Die Kouzeptsbücher. Da ich im Nachfolgenden noch ausführlicher über die Geschäftsgebahrung der Reichskanzlei, soweit sie mit der Anfertigung von Konzepten in Zusammenhang steht, sprechen will, soll von der Beschreibung des Konzeptsbücher hier nur so viel angeführt werden, als zum Verständnis des übrigen unumgänglich notwendig ist.

Von diesen im k. a. k. Haus-, Hof- und Staatsarchiv in Wien aufbewahrten Bänden ist der älteste, der mit IH bezeichnete. Er ist ein Papierband mit 379 foliirten Blättern. Auf dem Vorsteckblatt ist Folgendes in Zierschrift zu lesen: Diese registratur ist durch mich

1) Bresslau, Handb. d. Urkundenlehre 1, 744 und 754 .

2) Vgl. Lindner, Das Urkundenwesen Karls IV. S. 148 ff., ferner Seeliger, Die Registerführung S. 320 ff. und meine Ausführungen in Mon. palaeogr. XIII. 
Sixten Ölhafen secretarien, vor dem mein gnedigister herr von Mentz etc. churfürst die Romisch cantzley zu regiren angenomen hat, zu dem getrewesten meins vermugens zusamenbehalten und eingepunden der ku. $\mathrm{M}^{\mathrm{t}}$ und der canntzley zu eren und gut". Diese einleitenden Worte sind in mehrfacher Hinsicht von Interesse; einmal weil wir daraus ersehen wie weit man damals den Begriff "registratur" fasste, ist doch dieser Kodex eigentlich eine Sammlung von Kanzleikonzepten, die ohne gesichtet oder chronologisch geordnet worden zu sein, mit einigen Pergamentausfertigungen ${ }^{1}$ ) vereinigt worden sind, die aus irgend einem Grunde im Stadium der Expedition zurückgehalten wurden. Dann aber lässt sich insofern mit einiger Sicherheit der Zeitpunkt bestimmen, wann Bertold die tatsächliche Leitung der Reichskanzlei übernommen hat, denn nach dem Worten Ölhafens schloss er in diesem Augenblicke die Sammlung seiner Konzepte ab. Nun umfasst der Band Stücke vom Dezember 1493 (Bl. 170) bis Oktober 1494 (Bl. 19). Damit stimmen sowohl Beobachtungen überein, die wir in anderem Zusammenhange machen werden, als auch die Tatsache, dass auch die genannte "Ordnung" der Reichskanzlei den 3. Oktober 1494 als Datum trägt. Ferner ersieht ınan aus den Worten Ölhafens, dass die auf losen Bogen und Blättern gefertigten Konzepte jedenfalls bald nach Bertolds Amtsantritt zu dem vorliegenden Bande zusammengebunden wurden.

Schliesslich wäre noch zu bemerken, dass Band HH durchwegs deutsche Stücke enthält, die wohl in ihrer Mehrzahl von Sixtus Ölhafen geschrieben worden sind.

Ähnlich wie Band $\mathrm{HH}$ stellt sich ein anderer Papierband in die Reihe der Konzeptsbücher. Er trägt die Signatur Friedrich III.Max, gehört aber durchwegs der Kanzlei Maximilians an. Die irreführende Bezeichnung, die eigentlich einen ganz anderen Inbalt vermuten liesse, führt auf eine Verkennung des Kanzleigebrauches zurück. Wir werden späterhin darauf noch zu sprechen kommen.

Die 302 foliirten Blätter enthalten fast ausschliesslich Konzepte von deutschen Lehensurkunden, zwischen denen eingestreut verunglückte Pergamentmunda erscheinen (Bl. $82-85,109,130$ u. v. a.). Mit wenigen Ausnahmen aus dem Jahre 14932) datirt die Mehrzahl der Stücke von 1494. Die Zeitgrenzen bewegen sich zwischen 1493 Dezember und 1494 Juni 20 (Bl. 168, 215). - Eine sachliche oder zeitliche Anordnung lässt sich hiebei nicht entdecken. Wenig später als

1) B1. $215-123$.

2) Bl. 279, 280, 292 . 
die Vereinigung des ganzen Bandes scheint der Index angelegt worden zu sein.

Den beiden besprochenen Bänden ist es gemeinsam, dass sie noch vor Bertolds Antritt, also in der Hof kanzlei Maximiliaus entstanden sind. Aber auch die beiden nächstfolgenden Kodizes, die bereits in der Mainzer Kanzlei gesammelt worden sind, enthalten Stücke die vor der Amtsführung des Erzbischofs gefertigt worden sind.

Kod. JJ ist ein Papierband mit 294 foliirten Blättern im durchschnittlichen Ausmasse von $210 \times 310 \mathrm{~mm}$. Er enthält mit wenigen Ausnahmen ${ }^{1}$ ) lateinische Konzepte, die ursprünglich auf einzelne Bogen geschrieben ebenso wie bei den bereits besprochenen Bänden erst nachher zu einem Kodex vereinigt worden sind. Dies ist auch bei allen anderen Konzeptsbüchern der Fall. Desgleichen sei hier die für alle in Rede stehenden Kodizes geltende Regel erwähnt, dass nur die beschriebenen Blätter mit Zahlen versehen wurden. Die Zeit die JJ umfasst, reicht vom Herbst 1493 bis zum Herbst des Jahres 1495. Die in der Kanzlei entstandenen Stücke weisen im ganzen zwei Hände auf, von denen sich die eine als die des Sekretärs Virgilius Lunson bestimmen lässt. Ihm oblag das Konzipiren lateinischer Urkunden. Der zweite Schreiber dürfte in der Person seines Bruders Johannes zu suchen $\operatorname{sein}^{2}$ ).

Inhaltlich grenzt sich Band JJ von den anderen ähnlich Konzeptsbüchern dahin ab, dass er vornehmlich die Korrespondenz mit: dem Papst, den Kardinälen, den italienischen Fürstenhöfen, ferner Instruktionen $u$. dgl. enthält. Wie in den anderen Bänden ist auch hier eine gleichzeitige Anordnung zu finden. So steht auf dem Vorsteckblatte vermerkt: Expeditum Missiven et cetera id genus in Fủssen, dunn Bl. 8. Missive in Kempten, zwischen Bl. 24 und 25 Expeditum. anno 94. Missive et cetera id genus in Vienna, zwischen Bl. $30^{\circ}$ und: 37: Anno 95. Expeditum Missive et cetera id genus in Antwerpia et partibus iuferioribus, zwischen Bl. 107 und 108 anno etc. 95. Missive expedite Wormacie. Diese Vermerke, die entweder auf leergelassenen Blättern oder eingelegten Papierstreifen angebracht wurden, rühren vou der Hand Virgilius Lunsons her.

1) Bl. 29, 188.

2) Wenigstens findet sich Bl. $234 \mathrm{~b}$ das Konzept eines Briefes Virgilius Lunsons an seinen Vater, in welchem es heisst; „Preteritis Januarii mensis diebus venit ad me frater meus Jo. Lunson per litteras meas sepius antea accersitus in cancellariam Ro. regiam mihique in negotiis jam propter conventum principum congregatorum numerosis fideli auxilio fuit ". Der Brief datirt offenbar aus dem Jahre $\mathbf{1 4 9 5}$. 
Wie der eben besprochene Band gehört auch Kod. LL teilweise noch der Zeit vor Bertold an 1). Er ist ein Papierkodex der 180 foliirte und viele unbezeichnete Blätter im Durchschnittsformate $225 \times 325 \mathrm{~nm}$ umfasst. Eine gleichzeitige Notiz auf dem Vorsteckblatte gibt uns von vornherein Aufschluss über Inhalt und zeitliche Begrenzung. Da heisst es nämlich: Palatinatus et concessiones, doctoratus, dignitates date Lyntz anuo 93, Antwerpiensi 94, Mechlingensi 94, Iuspruck 94, Wormaciensi 95, Augustensi 96, Friburgensi 97, 98, Moguntinensi 99". Und wirklich reichen die Konzepte von 1493 Juni $27^{2}$ ) bis in den Herbst 1499.

Die hier vereinigten durchwegs in lateinischer Sprache abgefassten Konzepte sind, soweit sie überhaupt in der Reichskanzlei entstanden, fast insgesamt von dem obengenannten Sekretär Virgilius Lunson geschrieben. Unter die Konzepte sind nun auch Entwürfe von Briefen Bertolds $^{3}$ ) ja sogar soche Lunsons selbst ${ }^{4}$ ) geraten und nocb manches andere, wie unausgefertigte Pergamentmunda ${ }^{5}$ ) und eine Menge hübsch ausgeführter Wuppenbilder 6 ).

Eine bereits ursprüngliche Anordnung der Konzeple verraten ab und zu eingelegte Blätter, (ähnlich wie in JJ) auf denen, meist von Lunsons Hand, Ort und Jahreszahl verzeichnet stehen, wo und wann die nachfolgenden Stücke expedirt worden sind. So Bl. 51 tum Moguntie confirmaciones, investiture, preces et alia eorum genere anno 98 et 99 . Ähnlich firdet sich zwischen $\mathrm{Bl}$. 74 und 75 ein Papierblatt mit der Angabe: Confirmaciones, investiture, concessiones armorum et alia expedita Friburge anno etc. 98, dann zwischen Bl. 104 und 105: 1497 Expeditum Wormacie privilegium, commissiones, confirmaciones, investiture et cetera id genus, ferner zwischen Bl. 120 und 121 Expeditum zu Augsburg inverstiture, regalia et cetera id genus 96 und schliesslich zwischen Bl. 145 und 146: 1496 Expeditum Lyudaw confirmationes, investiture et cetera id genus. Unter die zwei letztei Abteilungen ist eine ganze Menge von Konzepten mit auderen Ausstellungsorten geraten.

Denselben Charakter wie die eben beschriebenen Konzeptsbücher trägt auch Band GG, ein Papierkodex mit 339 numerirten Blättern von verschiedenem Formate in der Durchschnittsgrösse von $230 \times 320 \mathrm{~mm}$.

1) Vgl. Monumenta palaeogr. XIII, 9.

2) LL Bl. 17-19.

8) Ebda Bl. 47, 52, 53', 61, 104 u. a.

4) Ebda Bl. 100, 101.

5) Fibda Bl. 70, 71.

6) Ebda Bl. $73^{a}, 74^{b}, 87^{a}, 88^{a}$ u. a. 
Er umfasst Konzepte in lateinischer Sprache abgefasster Urkunden. Instruktionen, offizieller Briefe nicht nur des Königs sondern auch von Kurfürsteu, ferner enthält er auch Stücke aus dem Einlauf ${ }^{1}$ ), Urkundungsbefehle ${ }^{2}$ ) und manches anderere ward hier zu diesem Buche zusammengebunden ${ }^{3}$ ). Die zeitlichen Grenzen sind 1494 April $\cdot 2^{4}$ ) und 1499 Juli $\mathbf{1}^{5}$ ).

Die Mehrzahl der kanzleigemässen Konzepte ist wie in JJ und LL von Virgilius Lunson geschrieben. Daneben kommt nur noch eine zweite Kanzleischrift vor. Auch hier wurden zwischen die Konzeptskonvolute, die aus Stücken von gleichen Ausstellungsorten gebildet wurden, Übersichtsblätter eingelegt, die in das Gewirr von Entwürfen wenigstens einige Ordnung bringen. So klebt schon auf dem Vorsteckblatte ein Längsstreifen, auf welchem, von Lunsons Hand vermerkt, zu lesen ist: Expeditum zu Worms inno 95 et Speier 94 confirmationes, investiture et cetera id genus" und zwischen Bl. 123 und 124 steht auf dem sonst unbeschriebenem Papierblatt: Privilegia, investiture et cetera id genus Antwerpie et Mechlinie expedita anno 94. Zwischen Bl. 155 und 156 liest mau: „Anno 94 Expeditum investiture et privilegia et cetera id genus in Ynnsprugk, zwischen Bl. 182 und 183: "Privilegia in Wienna expedita", dann Bl. 209a: Anno 94 Expeditum privilegia etc. in Kempten; zwischen Bl. 226 und 227 heisst es: „Expeditam privilegia investiture et confirmationes et cetera id genus in Fússen anno 94" und endlich auf Bl. $249 \mathrm{a}$ : „Sedecim littere et missive hic continentur et due sunt adjuncte 95, 96, 97, 98, 99". Von da an folgen bis zum Schlusse Konzepte aus den verschiedensten Ausstellungsorten, höchstens ab und zu nach Empfängern geordnet, wie zwischen Bl. 273 und 274, wo angemerkt wurde: „Negocia pro domino Urbano de Serralonga et marchione Montisferrati “ oder zwischen Bl. 304 und 305: „Ducem Saxonie tangentes" und auf Bl. 320 a: "In negotio Teutonicorum".

Was von den vorher behandelten Bänden gilt, hat auch auf $\mathrm{GG}$ Bezug. Man hielt sich nicht streng an diese Notizen, und manches Stück kum in eine Abteilung, in die es nicht gehört. Das letzte Blatt enthält ein in späterer Zeit angefertigtes alphabetisches Inhaltsverzeichnis.

1) GG. Bl, 15, 57, 66, 77, 121, 238 u. a.

2) Ebda z, B. Bl. 111'-112.

9) So z. B. ebda Bl. 208 eine Narrenkonfirmation oder s. g. Narrenwappen. Offenbar ein Faschingsulk übermütiger Kanzleischreiber.

4) Ebda Bl. 249.

5) Ebda Bl. 268. 
In der ehronologischen Reihenfolge schliesst sich nun Band KK an 1). Er umfasst 197 foliirte und viele nicht gezählte Blätter in einem Durchschnittsformate von $210 \times 310 \mathrm{~mm}$. Zeitlich gehören die hier gesammeiten in der Kanzlei angefertigten Konzepte der Periode 1496 Mai bis 1498 August an. Da es sich hiebei fast uur um lateinische Entwürfe von Missiven handelt, so dürfen wir annehmen, dass KK eigentlich bloss die Fortsetzung zum Bande JJ bildet, der wie bemerkt, mit 1495 abschliesst. Auffallend ist es, dass das vorliegende Buch eine ausuehmend grosse Auzahl von Stücken enthält, die schlechterdings mit einem Konzeptsbuche der Kanzlei nichts oder nur sehr wenig zu tun haben. Da findet sich eine recht erkleckliche Anzahl von Briefen des Erzbischofs Bertold von $\mathrm{Mainz}^{2}$ ), ferner ein Teil der Korrespondenz Virgilius Lunsons, sowohl Konzepte seiner.Briefe wie Originalschreiben an $\mathrm{ihn}^{3}$ ). Auch treffen wir in diesem Bande Suppliken, die an Maximilian ${ }^{4}$ ) und an Erzbischof Bertold ${ }^{5}$ ) gerichtet sind, ferner Urkundungsbefehle im Namen des Königs ${ }^{6}$ ), Kopien und Originale des Einlaufes ${ }^{7}$ ) und zum Schlusse gedruckte Urkunden ${ }^{8}$ ) aus der Zeit von 1495 August bis 149 ! Juli.

Von den in der Reichskanzlei gefertigten Konzepten sind auch in dem vorliegendem Kodex fast alle von Virgilius Lunson geschrieben, wie auch von dessen Hand wieder die Einteilungsblätter herrühren. Zwischen Bl. $29 \mathrm{a}$ und $29 \mathrm{~b}$ treffen wir ein Blatt mit dem Vermerke: „Expeditum zu Augsburg 96, Missive“, ferner zwischen Bl. 59 und 60: „1497 Expeditum Wormatie Missive“, dann liest man auf dem Blatte zwischen Bl. 73 und 74: „Expeditum Friburge 1498 Missive“ zwischen

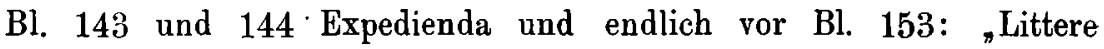
antique".

Mit Band KK schliesst die Reihe der Konzeptsbücher ab. Ich war bestrebt bei deren Beschreibung, mich auf das Notwendigste zu beschränken, um einerseits den nachfolgenden Ausführungen nicht vorzugreifen, anderseits Wiederholungen zu vermeiden. Es erübrigt mir nun noch einiges über den Charakter, die Entstehungsart und Geschichte dieser Bücher zu bemerken. Zwar schon durch die kurzen

\footnotetext{
1) Vgl. Die Beschreibung in Mon. palaeogr. XIII, 8.

2) $\mathrm{KK} \mathrm{Bl.} \mathrm{2,} \mathrm{3,} \mathrm{4,} \mathrm{6,} \mathrm{8,} \mathrm{24,} \mathrm{28,} \mathrm{29} \mathrm{b,} \mathrm{45,} \mathrm{92,} \mathrm{95} \mathrm{c,} \mathrm{104,} 131$ u. a.

s) Ébda Bl. $29 \mathrm{c}, 110,124 \mathrm{~b}$.

4) $\mathrm{KK}$ Bl. 10, $140 \mathrm{c}$.

5) Ebda Bl. 29 e, $29 \mathrm{f}, 79$ a.

f) Ebda Bl. 1, 291, 30 e, 9ృ c.

7) Ebda Bl. 183, 184.

s) Ebda Bl. 186-197; es sind im ganzen 12 Stücke.
} 
Hinweise bei Erwähnung der einzelnen Kodizes wurden gewisse Eigentümlichkeiten derselben hervorgekehrt, hier seien die Beobachtungen nun einheitlich zusammengefasst.

$\mathrm{Da}$ ist es zunächst die Anordnung und Aufeinanderfolge der verschiedenen Konzepte, die in den regelmässige Vermerken über Zeit und Ort der Expedition zu Tage traten. Gewiss waren dies Ansätze zur Entwirrung jenes Chaos von Kanzleiakten verschiedensten Charakters, aber trotzdem sind diese Bemerkungen weit entfernt davon, ein gewichtiger Nachschlagebehelf für den Benützer der Bücher zu sein. Finden sich doch z. B. Stuicke aus dem Jahre 1493 in nicht weniger als fünf Bänden, eingelegte Blätter mit den Angaben über die Expedition jedoch bezeichnender Weise erst von 1494 an, also erst mit dem Beginn der Kanzleiführung des Mainzers ${ }^{1}$ ). Aber auch dort wo man die Vermerke Lunsons antrifft, steht es mit der Ordnung nicht immer zum besten und man braucht eines beliebigen Band aufzuschlagen, um sich über den Mangel einer streng eingehaltenen Reihenfolge zu überzeugen ${ }^{2}$ ).

Auf den eingelegten Blättern und Streifen mit den Expeditionsangaben fiuden sich nicht selten Spuren von Siegelwachs, eine Erscheinung, die vielleicht damit zu erklären wäre, dass man jene Konzepte, die gemäss der Kanzleiordnung (I. 3) „nit noth weren zu registriern" sondern man "sunst verwaren" sollte, in Konvolute verpackte, bis man daran ging sie zusammenbinden zu lassen. $\mathrm{Ob}$ sie non bereits za Maximilians Zeit zu Büchern vereinigt wurden, lässt sich schwer entscheiden. Dies anzunehmen sind wir mit Sicherheit nur bei Band HH berechtigt, wo uns die Bemerkung Sixtus Ölhafens dies verbürgt. Anderseits steht fest, dess die besprochenen Bücher, ehe sie die modernen Pappeeinbände erhielten, fast durchwegs in Pergament gebunden waren ${ }^{3}$ ). Mit wie wenig Sorgfalt man bei der Herstellung der Kodizes vorging, beweist z. B. Band LL, der in verkehrter chronologischer Reihenfolge angeordnet ist.

1) Hiezu stimmt auch, dass der mit Friedrich III.-Max bezeichnete Kodex, wie auch Band $\mathrm{HH}$, welche wie gesagt vor Bertolds Amtsantritt entstanden, solche Einlageblätter mit den Expeditionsangaben vermissen lassen.

2) Ich greife ein Beispiel aus Band KK heraus. Dort steht zw. Bl. 29a und 29 b vermerkt: „Expeditum zu Augsburg 96, Missive , darauf folgen jedoch einige Briefe aus dem J. 1498, dann Bl. 29 h ein Brief aus dem J. 1496, Bl. 291 ein Urkundungsbefehl von 1498, Bl. $30 \mathrm{~b}$ wieder ein Brief an Bertold. Erst von Bl. 36 bis $\mathbf{5 2}$ reihen sich Urkundenkonzepte aus Augsburg an.

3) Wie aus den Verwaltungsakten des Wiener Staatsarchivs herrorgeht, wo sich der Bericht Rosenthals des ,Entdeckers der Reichsregistratur" vorfindet. 
Suchen wir nun für die ganze Reihe der Konzeptsbücher nach einem Einteilungsgrunde, so ergibt sich zunächst die Unterscheidung nach ihrer Herkunft und der Sprache, in der die darin befindlichen Stücke abgefasst erscheinen. Eigentümlicher Weise fallen hier beide Kriterien zusammen, denn die zwei Bände Kod. HH und Kod. „Friedrich III.-Max", die im Gegensatze zu den übrigen Büchern der Zeit vor der Kanzleiführung des Mainzers augehören, enthalten lediglich deutsche Stücke, während alle anderen dem lateinischen Expedite angehören. Eine Trennung, die hier mit seltener Konsequenz durchgeführt wurde, sich übrigens auch bereits in früheren Zeiten nachweisen lässt1).

Anderseits lässt sich, wenn auch nur andeutungsweise, eine Trennung nach Materien verfolgen. So enthält bekanntlich der Band „Friedrich III.-Max“ nur Lehensurkunden, JJ und KK hauptsächlich Missive und die übrigen Bände vorzugsweise Konzepte für Privilegien und Bestätigungen.

Schliesslich lässt sich die Frage nach der Herkunft der Konzeptsbücher nicht von der Hand weisen bezw, die Frage, ob wir es hier mit einer amtlichen oder privaten Sammlung zu tun haben. Ausdrücklich informirt darüber sind wir auch da nur in Bezug auf $\mathrm{HH}$, das seinen offiziellen Charakter durch die einleitenden Worte kund gibt. Man wird nun freilich in Analogie zu HH und in Hinblick auf die Kanzleiordnung, die ein Sammeln der Konzepte vorschreibt, anzunehmen haben, dass unsere Kodizes im Auttrage der Kanzlei angèlegt worden sind. Anderseits lässt sich jedoch nicht leugnen, dass es eine ganz bestimmte Persönlichkeit ist, der wir die Überlieferung dieser Bände verdanken. Wie ich schon herrorgehoben habe, sind uns aus der Mainzer Reichskanslei lediglich die Konzeptsbücher erhalten, die dem lateinischen Expedite entstammen, dagegen kein einziges deutsches.

In jener lateinischen Abteilung war nun Virgilius Lunson tätig. Die Spuren seines Fleisses konnten wir bei jeden der Bücher verfolgen, von seiner Hand rührt die Niederschrift der meisten Konzepte her und die Anordnung der Entwürfe nach der Expedition ist sein Werk. Leider hüllt sich seine Persönsichkeit in ein Dunkel, das ich trotz aller Bemühungen nicht recht habe lichten können. Jedenfalls war er ein gelehrter Mann, nicht eben alt, denn 1495 lebte noch sein Vater $^{2}$ ). Für seine Gelehrsamkeit spricht nicht nur das flüssige Latein,

1) Vgl. was Seeliger a. a. O. p. 352 t. gelegentlich der Ruprechtsregister ausführt, auch meine Ausführungen in Monum. palaeogr. XII, 1, , 3.

2) JJ Bl. 234' Brief Virgilius Lunsons an seinen Vater. 
das er schrieb, sondern auch die Tatsache, dass er 14981). (August 30 Freiburg) zum poeta laureatus ernannt wurde. Im übrigen weicht seine Stellung nicht wesentlich von dem Beamtentypus ab, der in jener Zeit eben aufkam und in allen Kanzleien zu finden war. Auf den unsicheren Ertrag der Sporteln angewiesen, war der Beamte zur Jagd nach Pfründen und Geschenken der Parteien genötigt2). Aus diesen Betrachtungen heraus wird man'manches erklärlich finden, was wir heutzutage als unverzeibliche Verstösse gegen die Kanzleiordnung zu bezeichnen geneigt wären.

Hielt nun Virgilius Lunson die Konzeptsbücher nicht in tadelloser Ordnung, so müssen wir doch sicher ihm das Verdienst zusprechen, für die Erhaltung dieser merkwürdigen Reste des Mainzer Kanzleiarchivs Dorsorge getroffen zu haben. Auch müssen wir in Betracht ziehen, dass aus der Abteilung für deutsche Ürkunden die gewiss viel reicheren Schätze an Konzepten - wenigstens aus der Zeit der Mainzer Kanzleiverwaltung - uns nichts bewahrt blieb. Insofern tritt zu dem gewiss offiziellen Charakter der Konzeptsbücher noch ein persönliches Moment, der Sammeleifer des Sekretär Lunson ${ }^{3}$ ).

Zeigten sich auch gerade unter Maximilian bemerkenswerte Ansätze, ein umfassendes Reichsarchiv zu begründen ${ }^{4}$ ), so blieb in der

2) LL Bl. 30 .

2) Lehrreich in dieser Beziehung ist ein Schreiben Urbanos de Serralonga, des Prokurators von Montferrat am königlichen Hofe, an Virgilius Lunson. Etwas ungeduldig über die verzögerte und schlechte Ausfertigung seines Pala. tinatbriefes schreibt er aus Casale ddo. 1496 Oktober 1. (LL Bl. 170): ,Presens nuncius dabit robis aparte florenos sex, ut habeatis causa faciendi mihi bonam expedicionem: Die pekuniäre lage Lunsons war übrigens wirklich keine glänzende, das erhellt aus einem Briefe, den er an Erzbischof Bertold rich. tete ( $\mathrm{KK} \mathrm{Bl} .110)$, worin er in beweglichen Worten den Kanzlei um Unterstützung anfleht, da er infolge einer Krankheit sich habe in Schnlden stürzen müssen, die ,bibalia, aber in der Kanzlei gegenwärtig so viel wie nichts eintrügen. Auch Erzbischof Bertold selbst war Geschenken nicht unzugänglich, so sandte ihm der oben erwähnte Serralonga, als er noch ein ganzes Jahr auf sein Privileg warten musste, duo pilei more Germanico videlicet unum de sirico cremessto alium de sirico nigro`. Vgl. hiezu noch Wagner, Nürnbergische Geheimschrift im 15. und zu Anfang des 16. Jahrh., Arch. Zeitsehr. 9. 18, .wo Bertold geradezı der Bestechlichkeit verdächtigt wird.

s) Wie schon erwähnt wurde, diente auch sein Bruder Johann in der Kanzlei. Er war, als er 1494 Februar 10 (GG Bl. 183) zum Sekretär Kapellan und continuuis commensalis von Maximilian ernannt wurde, bereits, rector parrochialis ecclesie in Keutzingen`. 1495 erwähnt dann Virgil in dem Brief an seinen Vater: Frater meus optima prebenda in kathedrali ecclesia Spirensi provisus jam dudum possessionem acceptare decrevit. Später. war er auch in diplomatischen Diensten des Königs in Italien. Vgl. meine Ausführungen in Mon. Palaeogr. XIII. 8.

4) Vgl. A. Tille in Mitteil. des Instituts f. österr. Geschichtsf. 22, $296 \mathrm{f}$. 
Praxis doch die Übung $z u$ recht bestehen, dass die einzelnen Beaniten das Kanzleimaterial förmlich als ihr Privatgut betrachteten. Hiefür ist uns aus einer nur um ein Vierteljahrhundert späteren Epoche ein klarer Beleg erhalten, der mir umsomehr für die Erkenntnis der Zustände in der Mainzer Kanzlei lehrreich erscheint, als er auch auf diese frühere Zeit zurückgreift. Anlässlich eines Reichskammergerichtsprozesses, bei dem es sich um die von Frankreich okkupirten Reichslehen handelte, schrieben nämilich die Räte des Esslinger Reichsregimentes an Erzherzog Ferdinand (1524 Oktober 15) ${ }^{1}$ ), es wären ihnen vor allem die Urkunden und Investitursreversale nötig, um die Eigentumsrechte des Reiches auf die Dauphinée etc. zu erweisen. Sie bitten deshalb den Erzherzog, in den Schriften und Briefen Friedrichs III. und Maximilians I. in Wien und Innsbruck nach solchen Stückeu nachforschen zu lassen, ebenso bei deren alten Sekretären bezw. bei deren Nachlass, wie bei Stürzel, Sernteiner, Ziegler, Renner u. a. Gleichzeitig teilen sie mit, dass sie an den Mainzer als Erzkanzler des Reiches und den Erzbischof von Trier als den Galliens schrieben, da die in Worms vom Sekretär Spiegel über Regalien, Lehen und Bestätigungen etc. gefertigten lateinischen Minuten und Konzepte trotz Reichsbeschlusses noch nicht an sie gesendet worden $\operatorname{sind}^{2}$ ).

In Anbetracht dieser von Kanzlei zu Kanzlei sich fortschleppenden Nachlässigkeit wird man das Verdienst unseres Lunson umso höher einschätzen müssen.

Die Konzeptsbücher blieben wie die ganze ältere Reichsregistratur durch die Fürsorge der tirolisehen Linie der Habsburger uns erhalten. Wie wir wissen ${ }^{3}$ ), erkannte man in Innsbruck bald deren praktischen Wert. Im Jahre 1750 ward V. Rosenthal durch eine Notiz des Reichshofratspräsidenten, Grafen Wurmbrand, aus denı Jahre 1736, die er in einem alten Schatzgewölbe-Repertorium fand, aufmerksam gemacht, dass sich die Reichsregisterbände vermutlich in Innsbruck vorfinden dürften. Daraufhin wandte sich dieser tatkräftige Maun an den Direktorialpräsidenten von Haugwitz und erwirkte sich die Vollmacht, die wertvollen Kodizes nach Wien bringen zu dürfen ${ }^{4}$ ). Unter ihnen

1) Gedruckt bei Chmel Notizenblatt 2, $247 \mathrm{ff}$.

2) Übrigens bedurfte es nach dem 'Tode Waldners erst eines ausdrücklichen Befehls des Kaisers, um aus dessen Nachlass einige , Freiheitsbriefe des Hanses Österreich " herauszubekommen. S. Adler, Die Organisation der Zentralverwaltung (Leipzig 1886) S. 437.

3) Seeliger, Registerführung S. $245 \mathrm{f}$.

4) Ich glaubte dies hier anführen zu dürfen, weil hiedurch sowohl Schönherrs Angaben Archiv. Zeitschr. 11, 115 ff. und Mitteil. der Archivsektion der Zentralkomm. 2, 161) als auch die Seeligers ergänzt werden. Die Eingaben, 
befanden sich, wie es scheint von jeher, auch die Konzeptsbücher, obwohl sie mit Registerbänden nur wenig gemeinsam haben.

2. Das Konzept. Ohne Zweifel wäre unsere Kenntnis des Kanzleiwesens früherer Epochen eine viel eindringendere, stünden uns aus jenen Zeiten die Entwürfe der ausgestellten Urkunden zur Verfügung. Schon die Tilgung des einen oder anderen Passus, die Anläufe zu einer bestimmten Formel, die man im letzten Augenblicke zu unterdrücken gut fand, liessen uns in die Intentionen des Ausstellers tiefer blicken, als die glatte, kalligraphisch vollendete Reinschrift. Aber auch die formale Betrachtung des Werdeganges einer Urkunde käme bei Untersuchung des Konzeptes zu seiner Geltung. Der Unterschied etwa der Schrift des Konzipisten gegenüber der des Korrektors würde uns über die Anteilnahme der verschiedenen Beamten an der Entstehung des Diplomes belehren - aber von all dem finden sich eben in der Geschichte des älteren Urkundenwesens kaum leise Andeutungen. Ja man ist für jene Perioden vielfach in Zweifel, ob nicht in der Regel der Diktator mit Umgehung jeglichen Entwurfes überhaupt sofort ins Reine schrieb 1 ).

Für die Zeit Maximilians I. nun lassen sich alle diese Fragen wesentlich einfacher beantworten als selbst noch für die Periode der Luxemburger und Friedrichs III.

Will eine Partei ein neues Recht sich erwerben oder für ein bereits besessenes eine Bestätigung erhalten, so reicht sie eine Supplik ein. In den Konzeptbüchern sind uns verschiedene solche Bittschreiben erhalten. Vergleicht man sie miteinander, so findet man, dass sie recht individuell abgefasst sind und nicht etwa, wie man glauben könnte, auf ein bestimmtes einheitliches Formular zurückgehen. Während etwa einmal ein Mönch dem König verspricht, dreissig Messen zu lesen, wenn er seinem Kloster die Privilegien bestätigt und in langatmigen Ausführungen seine Verdienste mit konventioneller Beteuerung der eigenen Unwürdigkeit vermischt ${ }^{2}$ ), finden sich anderseits Suppliken, die sich auf eine trockene Aufzählung der Bitten ohne jegliche Floskeln beschränken, so geschäftsmässig und formlos, dass man sich dies nur erklären kann, wenn man annimmt, dass diese Bittschriften in den seltensten Fällen dem König wirklich vorgelegt worden sind. Deshalb

Memoriale und Verzeichnisse über den Auslieferungsvorgang befinden sich in den Verwaltungsakten des H.-, H.- und St.-A. in Wien.

1) Bresslau, Urkundenlehre S. 751, scheint mir in dieser Hinsicht etwas zu weit zu gehen.

2) GG Bl. 16. 
wandte man sich vielfach gleich direkt an den Erzbischof von Mainz ${ }^{1}$, ja oft nicht einmal an diesen, sondern an andere einflussreiche Beamte ${ }^{2}$ ).

Über die Behandlung der Suppliken besagt die Kanzleiordnung von 1494 nichts, aus dem einfachen Grund, weil die Reichskanzlei ja fern vom Hofe war und sie mit der Erledigung der eingelaufenen Gesuche zunächst wenigstens nichts zu tun hatte. Diesen Mangel ersetzt uns ejnigermassen der Entwurf einer Instruktion für den Hofkanzler ${ }^{3}$, der aus der Zeit ron 1497-1498 stammen dürfte. Danach hat der Hofkanzler die "sanntbrief" (wohl Missire) und Supplikationen und alle anderen Schriften, die ihm überantwortet wurden, in den Rat zu bringen. Er hat dann mit eigener Hand den "ratslag“ darauf zu schreiben und den Sekretären zu befehlen, die Briefe danach zu verfertigen. Erst der Rat entscheidet, welche Dinge vor den König gebracht werden sollen und welche nicht.

Natürlich kann dieser Instruktionsentwurf nur als Analogon herangezogen werden ${ }^{4}$ ), aber ähnlich ist die Geschäftsgebahrung in Reichssachen jedenfalls auch gewesen. Auch hier oblag es zunächst den verschiedenen Räten, die eingelaufenen Bittschriften zu überprüfen. Sie legten dem Könige wohl nur Auszüge vor. Gab er seine Einwilligung, so übermittelten sie die Akten der Reichskanżlei.

Nach welchen Gesichtspunkten sich nun die Räte in die Fülle des Stoffes teilten, ist im genaueren leider nicht festzustellen. Für uns, die wir nur die lateinischen Konzeptsregister zur Verfügung haben, tritt Ludovicus Brunus in den Vordergrund. Selbst Italiener hatte er die italienischen Angelegenheiten zu leiten. Neben ihm treffen wir auch Nikolaus Ziegler, der ja nach dem Ende der Mainzer-Kanzlei die Reichssachen zu versehen hatte ${ }^{5}$ ). Einmal begegnet uns der auch sonst in Maximilians Diensten viel beschäftigte Marquard Breisacher $\left.{ }^{6}\right)$.

1) Z. B. KK Bl. 29 e, 29 f, 79 a; LL Bl. 283. u. a.

2) So richtet Abt Bernhard von Stams seine Beschwerde bezw. Supplik an Konrad Stürtzel, Niklas von Firmian. und Florian Waldauf, ohne dass einer dieser drei in irgend einem Zusammenhang zur Reichskanzlei gestanden wäre.

9) Abgedr. bei Adler a. a. O. S. 511 ergänzt von V. v. Kraus, Itinerarium Maximiliani I., Archiv f. österr. Gesch. 87, 251 f.

4) Der Unterschied zeigt sich sowohl in den Bestimmungen über die Kanzleiunterfertigungen als auch über das Registerwesen. V. v. Kraus a. a. O. S. $251 \mathrm{ff}$. macht in seinen Ausführungen gar keinen Unterschied zwischen Hof- und Reichskanzlei.

5) LL zw. Bl. 102 und 103 ist eine Zuschrift Nikolaus Zieglers an Erzbischof Bertold erhalten des Inhalts: Gnedigster herr! Die k. Mt. hat Alexius Tanel ein caplanatbrief bei. E. F. G. zu machen verschafft, als dann E. F. G. wol zu thun weiß. E. F. G. underteniger N. Ziegler.

6) JJ zwischen Bl. 96-97 findet sich folgendes Schreiben: Domine can- 
Ausserdem sandte der König - wie es scheint, besonders su Zeiten der Spannung zwischen ihm und dem Kanzler - an Bertold förmliche Orkundungsbefehle, die mit ad mandatum domini regis proprium $^{1}$ ) oder mit per regem per se unterfertigt sind, wobei sich auch Matthäus Lang unterzeichnet ${ }^{2}$ ).

Dass aber der Geschäftsgang bei gewöhnlichen Suppliken über Reichssachen ähnlich war, wie er in der Instruktion für den Hofkanzler, geschildert wird, zeigt sich auch darin, dass tatsächlich auf mancher dieser Bittschriften sich Vermerke vorfinden, die wohl ron dem Rate herrühren, der gerade darüber das Referat hatte. So steht einmal unter einem Gesuche um Ernennung zum comes palatinus "fiat pro illis tantum, et non pro descendentibus" ${ }^{3}$ ) oder unter einer Supplik Lukas Fuggers: „fiat den solchs auf sein costen zu bevelhen und credentz zu geben ${ }^{4}$ ). Ein andermal wurde auf dem Schreiben des Elekten von Toul um Regalienverleihung ein umständliches Gutachten der Räte vermerkt $\left.{ }^{5}\right)$.

Ganz anders wickelte sich natürlich der Vorgang ab, wenn es sich nicht um Urkunden handelte sondern um den offiziellen Verkehr mit auswärtigen Potentaten oder Staaten. Hier entfällt selbstverständlich die Supplik und wird unsomehr Sorgfalt auf die Abfassung des Konzeptes verwendet. Vereinzelt überliess es freilich - wohl nus politischen Gründen - der König dem Mainzer oder den Reichs-

cellarie. De mandato sermi d. Ro. regis oportet expedire infrascripta pro oratoribus in Scociam transmittenda et celeriter: credentiales ..... Item copias illarum litterarum, quomodo sermus d. Ro. rex scribet principibus eeclesiasticis et secularibus imperii et quomodo judicio sue $\mathrm{m}^{\text {tis }}$ ipsi subditis et incolis suis scribere debeant. Item etiam de mandato regis debent ipse littere ad principes et episcopos scribi et ingrossari in forma.

M. Breisacher.

1) KK Bl. 1 und 31 .

2) Z. B. KK Bl. 29 l. Orig. pap. rote Siegelspur. Die Zuschrift lautet wört. lich: Maximilian von gots gnaden Romischer Kunig etc. Erwirdiger lieber neve und churfurst. Wir empfehlen D. L., das Du den von Metz auf unnsern briefe, so sy Dir furpringen werden, gnugsam quittung, wie sich gepúrt, fertigst und solchs nit lassest. Daran tust Du unnser ernstliche meynung. Geben zu Metz an pfintztag nach Michaelis anno etc. IXXXXVIII unnsers reichs im dreyzehenden jar

per regem per se

M. Lanng.

(Rückwärts die Adresse.)

9) LL Bl. 32.

4) $\mathrm{KK} \mathrm{Bl.} 140 \mathrm{c}$.

5) JJ B. 202: Consultamus revmum dominum electum per regiam mtem sub imperii concordatis defendi in possessione debere pro justicie tenore prefatumque electum sub ccncordatis comprehendi eo, quia sit in collegio cathedralis ecclesie, que immediate imperio est suhjecta canonice electus. 
ständen, einen an ihn gerichteten Brief zu beantworten 1 ). Anderseits wickelte sich das Orkundengeschäft vielfach in seinen ersten Phasen am königlichen Hofe ab, denn die weite Entfernung der Reichskanzlei machte dies oft aus praktischen Gründen notwendiga ${ }^{2}$ ). Die Trennung dieses Amtes vom Sitze des Königs war in jeder Beziehung einer raschen Ausführung hinderlich. Die von der Reichskanzlei eingesandten Konzepte genügten nicht immer den Ansprüchen, die man am Hofe erfüllt wissen wollte. Nun war es erst Sache des betreffenden Rates den Entwurf zu prüfen, eventuell zu verbessern ${ }^{3}$ ). Es kam aber vor, dass man mit der Fassung eines Konzeptes überhaupt nicht zufrieden war und wüuschte, dass über denselben Gegenstand ein zweites angefertigt werde. So bei einem Schreiben Maximilians an den Papst, in dem er dagegen protestirt, dass Kölner Bürger vor die Kurie zitirt wurden. Unter dem von Lunson geschriebenen Konzept steht von anderer Hand: Prestme Domine. Rogo, ut talem copiam emendetis, ex qua tantum sensum materie intelligetis et melioribus sententiis et verbis copiosioribus articulis pro conservandis sacri imperii Romani juribus. Eliminatam et perfectam rogo hac nocte remittatis ${ }^{4}$ ).

War es auch, wie wir gesehen haben, zumeist Aufgabe der Räte, die von der Reichskanzlei gelieferten Konzepte durchzusehen oder zu verbessern, so geschah es doch, dass der König selbst in das Urkundungsgeschäft persönlich eingriff ${ }^{5}$ ). Doch handelte es sich hiebei natürlich vor allem um Stücke, die politischen Charakter tragen.

Wir haben der Supplik bereits Erwähnung getan. Galt es nun für eine Partei, die Verbriefung alter Rechte und Privilegien zu er-

1) KK als Vorsteckblatt des Originalschreibens des Königs von Polen an Maximilian d. d. 1496 September 16; Maximilian sendet nun diesen Brief an Erabischof Bertold und überlässt es diesem und den zu Lindau versammelten Ständen, die Antwort zu fertigen. (Maximilian an Erzbischof Bertold d. d. 1496, November 11. ebda Bl. 1 a). Während der König gar nicht den Reichstag besucht, wird doch in seinem Nanem die Antwort an Johann Albrecht von Polen unter dem Datum 1496 Dezember 1 Linciau abgesandt.

2) GG Bl. 261. Ein in der Hofkanzlei gefertigtes Konzept über Reichs. sachen. Darunter steht: Lieber herr canzler, wellet dise copi ubersehen und dem Virgili die brief darauf, als er waiss ver[richten] lassen. Sernteiner.

s) So schreibt Ludovicus Brunus in seinem interessanten Briefe an Erzbischof Bertold: Mitto igitur Vre illme D. litteras illas, quas legeram, c orrectas per me secundum mandatum Vre revme Pattis et consilii. JJ Bl. 249.

4) JJ Bl. 232.

5) So heisst es JJ Bl. 226 auf einem Konzept oben: Visum et lectum per regem, et sie jussit emendari et corrigi und ebenda $\mathrm{Bl}$. 229 visum et lectum per regem, ein andermal ebenda $\mathrm{Bl} .275$ noluit recipere und Bl. 276 Rex noluit ita admittere. 
langen, so musste sie hiefür natürlich der Kanzlei die betreffenden Vorurkunden liefern. In welcher Weise dies geschah, lässt sich auf Grund unseres Materials nicht sicher entscheiden. Dort, wo wir Kopien antreffen, die mit einer notariellen Beglaubigung versehen sind ${ }^{1}$ ), beantwortet sich die Frage von selbst. Nun finden sich in den Konzeptsbüchern einzelne Blätter und ganze Hefte, die durch Scbrift und äussere Form sich deutlich von dem Kanzleimaterial unterscheiden. Sie enthalten Abschriften der Privilegien, welche man bestätigt haben wollte, doch tragen sie kein amtliches Visum. Es liesse sich denken, dass man die Originale samt den Kopien einsandte, sie in der Reichskanzlei verglich und letztere daselbst zurück liess. Doch finden sich für diese Vermutung weder in den Konzepten noch in den Kopien Anhaltspunkte ${ }^{2}$ ).

In welcher Weise die Vorurkunden für das Konzept verwendet worden sind, dafür kann Monum. palaeogr. III, 9 a als typisches Beispiel gelten. Die zu inserirende Urkunde wird nicht eigens abgeschrieben sondern vur durch die Anfangsworte angedeutet. Sonst gilt in der Reichskanzlei die Regel, dass selbst für häufig vorkommende Urkundenarten eigens Konzepte angefertigt wurden $\left.{ }^{3}\right)$.

Der Weg von der Supplik bis zur Niederschrift des Entwurfes bedeutete nur das erste Stadium der Gesehichte des Konzeptes. In der vielzitirten Kanzleiordnung I. 1 heisst es ausdrücklich, dass die Minuten, bevor sie ingrossirt werden, dem Erzkanzler oder dessen Stellvertreter übergeben und von diesem subskribirt werden müssen. Wenn wir nun das oben erwähnte Faksimile darauf hin prüfen, bemerken wir rechts unten, knapp unter dem Text ein „bs $\beta^{t}$, das in seiner charakteristischen Form sich auf der Mehrzahl der Konzepte vorfindet. Diese Signatur ist unzweifelhaft in "bertoldus subscripsit" aufzulösen. Sie wurde von dem Erzbischof eigenhändig beigefügt. Ihre Eigenhändigkeit geht auch aus dem Vergleiche mit einigen Originalen hervor, die die Unterfertigung des Erzkanzlers tragen ${ }^{4}$ ). Die Frage, zu welchem Zeitpunkte diese Sub-

1) Z. B. GG Bl. 163 a, KK Bl. 183, LL Bl. 70, 109 u. a.

2) Die Tatsache, dass in den Registern $X 1$ und $X 2$ bei inserirten Urkunden Nachzeichnungen von Monogrammen sich finden, könnte die Meinung auf kommen lassen, dass in diesen Fällen die. Originale der Vorurkunden vorgelegen seien. Es wäre ja möglich, doch treffen wir derartige Nachzeichnungen bereits in eingesandten Kopien z. B. LL Bl, 160, HH Bl. 365, KK Bl. 195.

s) Vgl. Bresslau a. a. O. 755 .

1) H.-, H.- und St.-A. in Wien: 1494 Juni 28; 1495 Juni 18; 1500 Juli 28. Bei allen diesen Urkunden zeigt sich in der Unterfertigung Bertolds das charakterische Minuskel b. - Aus der Zeit vor Bertolds Kanzleiführung dagegen findet sich auf Konzepten nirgends ein ähnlicher Vermerk. 
skription erfolgte, lässt sich dahin beantworten, dass sie in der Regel vor der Datirung geschah. Dies beweist der Umstand, dass viele Konzepte überhaupt keine Zeitangabe aufweisen und trotzdem mit dem Subskriptionszeichen Bertolds regelrecht versehen sind ${ }^{1}$ ); nicht selten bemerkt man - wie auch an unserem Faksimile - dass das Datum der Unterschrift ausweicht'2). Überhaupt gilt auch für die Reichskanzlei, was Ficker im allgemeinen annahm ${ }^{3}$ ), dass nämlich die Konzepte zunächst keine Datirung erhielten. Dies ergibt sich nicht nur aus obiger Betrachtung sondern auch aus der Tatsache, dass die Schriftzüge und Tinte des Datums in vielen Fällen erkennen lassem, dass dieses erst später hinzngefügt wurde ${ }^{4}$ ).

War die Signirung durch den Erzkanzler erst unter Erzbischof Bertold eingeführt worden, so bestand der Gebrauch, bei den Konzepten oben links den Expeditionsvermerk anzubringen, schon lange vorher. Dies können wir z. B. in Band HH konstatiren, der noch der Kanzleitätigkeit des Sixtus Olhafen angehört, und ebenso im Bande „Friedrich III.-Max I."

Stücke, die aus irgend einem Grunde in der Kanzlei zurückbehalten wurden, machte man meist durch ein "non est expeditum“ kenntlich5). Auch da lässt sich die grosse Anteilnahme bemerken, die der Mainzer persönlich selbst an den Details des Urkundungsgeschäftes bewies. Dies bezeugen Notizen wie: Visum et lectum per revmum et sic jussum expediri ${ }^{6}$ ) oder ähnlichen Inhalts ${ }^{7}$ ). Als aber z. B. einmal ein Stück nicht von der Reichskanzlei expedirt wurde, verzeichnete man sofort: Expeditum sed non in nostra cancellaria $\left.{ }^{8}\right)$. Bertold unterliess

1) Z. B. GG Bl. 266, JJ Bl. 47, 48, 55, 109 etc. KK Bl. 81; Bei GG Bl. 263 steht das bs $\beta^{t}$ vor den Zeugen. Auf GG Bl. Bl. 121 hat Bertold selbst das Datum auf das Konzept gesetzi. - Ein interessantes Analogon zu diesem Geschäftsmodus bietet sich uns übrigens aus der Kanzlei Karls IV. Vgl. Steinherz Mitt. des Instituts f. österr. Geschichtsf. 9. S. $616 \mathrm{f}$. Wir sehen, dass auch dort die Konzepte erst von einem höheren Beamten durchkorrigirt und von einem dritten mit dem Unterfertigungsvermerk versehen wurden. Erst nachdem dies erfolgt war, wurden die Zeugen und die Datirung hinzugefügt.

2) Z. B. GG Bl. 292; JJ Bl. 37, 41, 49 etc.; KK Bl. 1, 23, 35 etc. LL Bl. 26, 45, 92 etc.

3) Beitr. z. Urkundenlehre $\S 191$.

4) Z. B. GG Bl. 301 ; JJ Bl. 41; KK Bl. 1, 35, 37, 43 etc.; LL EI. 35 etc.

5) Z. B. JJ Bl. 245, 248; KK Bl. 81, 134 b; LL Bl. 147, 178; einmal findet man auch die Bemerkung nondum est expeditum GG Bl. 111.

6) KK Bl. 130.

7) Presens copia admissa per revmum $d$, archiepiscopum et jussa expediri LL 65; Visum et lectum per revmum $d$, archiepiscopum Moguntinensem et sic jussit expediri LL 93 ähnlich ebda 97 .

8) LL Bl. 37. 
es auch, dieses Konzept zu signiren. Die Rivalität zwischen Hof- und Reichskanzlei gefiel sich selbst in dem Festhalten an Kleinigkeiten ${ }^{1}$ ).

Das Verhältnis zwischen Expedition und Datum wird dahin aufzufassen sein, dass wohl in der Regel die Datirung den Tag der Expedition bezeichnet. Dies scheint mir auch aus folgender Bemerkung hervorzugehen, die sich unter einem Konzept vermerkt vorfiudet: fiant litere duplicate propter locorum distantiam mutatis mutandis et mutatis verbis sed servatis sententiis et prime litere datentur sub die expeditionis secunde circa finem novembris et date signentur extra ipsas literas vel sub eorum operculo, ne ordo confundatur et fiant copie ${ }^{2}$ ).

Nebenbei sei erwähnt, dass die Verzögerung des Expedits Grund zu ständigen Klagen der Trkundenempfänger gab und von den schlechtbezahlten Kanzleibeamten, wie es scheint, dazu benutzt wurde, von zahlungskräftigen Parteien bibalia herauszupressen ${ }^{3}$ ). Bei wichtigen Angelegenheiten mussten die königlichen Räte die Beamten der Reichskanzlei stets erst ermahnen, die Ausfertigung möglichist zu beschleunigen ${ }^{4}$ ). Wie langsam mag $d a$ der amtliche Apparat in Fällen von geringerer Bedeutung funktionirt haben.

Ich glaube im Vorhergehenden die wesentlichen Punkte angeführt zu haben, die für die Geschäftsgebahrung des Konzipisten in der deutschen Reichıskanzlei in Betracht kamen; aber meine Darstellung würde eine Lücke aufweisen, wollte ich mich mit dem Gesagten begnügen. Gerade die, wie ich glaube, interessanteste Erscheinung auf dem Gebiete des Konzeptswesens wurde bisher noch nicht besprochen und sie bedarf umso dringender der Erwähnung, als ibre Kenntnis in manchen Dingen eine erwünschte Handhabe zur Kritik einzelner Urkunden bieten dürfte.

Wir haben bereits bemerkt, dass es von Seite der Parteien Gepllogenheit war, Kopien der zu bestätigenden Urkunden an die Kanzlei

1) Vgl. H. Ulmann, Kaiser Maximilian I, S. 293, A. 2.

2) KK Bl. 17 ; ebda Bl. 40 steht auf einem Konzept: Item den ersten brieff sol man das datum geben auff den tag, so er geschriben wirt, item den andern das datum aufi unser frawentag in dem monat septembris.

s) Vgl. S. 267 den Brief Serralongas an Virgilius Lunson ferner LL Bl. 177' schreibt derselbe Serralonga an den Mainzer: Mitto Revme D. V. tacxam factam illarum expedicionum, quas mecum portavi, videlicit florenos centum renenses et bibalia pro scriptoribus et canzelaria florenos viginti.

4) Wegen Erteilung eines Wappenbriefes für den Orator der. Stadt Siena schreibt Ludoricus Brunus an die Kanzlei: et hoc jubet rex statim litteras expediri, quia in omnibus aliis idem orator est expeditus. "Dixi hoc revmo d. Moguntino, qui jubet per magum Virgilium rem expediri. Rogo cito fiat, quia instat ipse apud me. GG Bl. 111 a. Ähnlich schreibt Marquard Breisacher an die Reichskanzlei JJ Bl. 96 f. 
zu senden; ja es hat fast den Anschein, dass man sich selbst mit unbeglaubigten Abschriften begnügte. Sei dem nun, wie ihm wolle, Tatsache ist es jedenfalls, dass dieser Gebrauch wenigstens in einem Falle zư regelmässigen Übung geworden zu sein scheint, nämlich bei Belehnungen. Es war ja nichts natürlicher, als dass diejenigen, die beim Regierungsantritte Maximilians um ihre Belehnung einkamen, kurzer Hand eine Kopie der betreffenden Urkunde, die ihnen einst Friedrich III. verlieh, einsandten. Da bedurfte es denn wohl auch keiner notariellen oder anderweitigen Beglaubigung, da man den Text mit den damals jedenfalls noch vollständig erhaltenen Lehensregistern vergleichen konnte ${ }^{1}$ ).

Statt nun ein neues Konzept anzufertigen, tilgte man einfach den Titel durch Unterstreichen, schrieb den Namen des Königs dariiber und änderte alle auf das Kaisertum Friedrich III. sich beziehenden Formeln entsprechend um. Das alte Datum wurde ebenso wie der Titel unterstrichen und nach erfolgter Kanzlersubskription das neue hinzugefügt. Dieser Vorgang war so regelmässig, dass eines unserer Konzeptsbücher ihm seine Signatur verdankt. Es ist dies der mit „Friedrich IIJ.-Max" bezeichnete Kodex, dem man in Unkenntnis darüber, welche Bewandtnis es mit den vielen Kopien von Friedrichsurkunden habe, diesen unzutreffenden Namen gab. Aber auch in den anderen Bänden sind ähnliche Stücke verstreut ${ }^{2}$ ). Ich habe ein solches aus LL Bl. 144 in den Monumenta palaeographica XIII $9 \mathrm{~b}$ als Faksimile veröffentlicht und auch auf palaeographischem Wege nachweisen können, dass es sich hier um eine Kopie einer Urkunde Friedrichs III. handelt, die in der Kanzlei des Empfängers, des Bischofs von Lüttich, niedergeschrieben worden sein dürfte. Die Überschrift: Forma regalium mutatis mutandis pro episcopo Leodiensi beweist deutlich, zu welchem Zwecke man die Abschrift anfertigte und dass man sich desselben wohl bewusst war.

Pflegte man der Supplik die Kopien jener Urkunden beizulegen, deren Bestätigung man zu erlangen wünschte, so war es nur ein kleiner Schritt weiter, wenn man auch gleich selbst die Bestätigungsurkunde konzipirte, zumal man aus der Erfahrung bemerken konnte, wie eng meist das Formular des neuen Diploms sich an das des Vorgelegten anlehnte. Bei den Lehensbriefen sprang das vielleicht noch mehr in die Augen. Aber der grösste Antrieb, zur Fertigung eines Konzeptes

i) Vgl. Seeliger, Registerfübrung S. 302.

2) GG Bl. 169; HH Bl. 71, 203, 228 etc. Übrigens findet sich auch eine amtlich beglaubigte Kopie einer Urkunde Friedrichs (GG Bl. 37), die in der Kanzlei als Konzept verwendet worden ist. 
mag für die Parteien dort bestanden baben, wo sie mit der Fassung der alten Urkunde nicht ganz einverstanden waren oder eine Erweiterung ihrer Rechte wünschten. So heisst es einmal auf einem von der Stadt Kolmar eingesandten Entwurf ${ }^{1}$ ): Wie wir uff dis mol gern die Confirmatz hetten.

Diese eingesandten Konzepte waren selbstverständlich von verschiedenem Werte, je nachdem ihr Verfasser das Kanzleiformular beherrschte oder nicht. Im besten Falle brauchte der Beamte unter ein solches Stück, nachdem es vom Kanzler signirt worden war, nur das Datum anzubringen, um es ingrossiren zu lassen. Meist stellte sich aber die. Notwendigkeit heraus Verbesserungen an dem Entwurf der Partei vorzunehmen, wissen wir doch, dass Bertold streng darauf sah dass der "stilus der cantzley" eingehalten werde ${ }^{2}$ ). So verbesserte man in einem für Trier eingelaufenem Konzepte ${ }^{3}$ ) reverendus pater in venerabilis pater, consanguinei in nepotis u. s. W. oder in einem anderen, das der Kanzlei des Markgrafen von Montferrat entstammte, die Poenformel, die mit Si quis auten begann in die mit Nulli ergo omnino etc. ${ }^{4}$ )

Solche Fälle waren immerhin noch günstig, manchmal sandte man aber an die Kanzlei ganz phantastisch abgefasste Vorlagen, so dass der Sekretär gezwungen war, erst recht noch ein Konzept anzufertigen, das die sachlichen Momente des eingelaufenen Entwurfes verwertete 5 ).

In allen jenen Konzeptbüchern, die sich nicht auf Stücke beschränken, die lediglich den diplomatischen Verkehr mit dem Auslande betreffen, finden sich solche von Empfängern gefertigte und geschriebene Urkundenentwürfe in grosser Zahl. Diese Erscheinung ist ungemein überraschend, aber keineswegs neu. In dem höchst instruktiven Aufsatze Tangls: „Der Entwurf einer Königsurkunde aus Karolingerzeit"6) wird der Nachweis erbracht, dass ein vermutlich vom Abt-Bischof Salomon angefertigtes Konzept unmittelbar. Vorlage für

1) $\mathrm{HH} \mathrm{Bl.} \mathrm{191'.}$

2) Seeliger, Kanzleiordnung IV. 19.

3) GG Bl. 81 .

4) GG Bl. 59 ff.

5) So sandte ein Mönch namens Joh. Ortenberg aus dem Kl. Neuhausen nebst einer langatmigen Supplik ( $G G$ Bl. 16) das Kozept der auszustellenden Bestätigungsurkunde für sein Kloster ein. Ganz gegen das Herkommen beginnt sein Entwurf mit einer Invocatio: In nomine sancte et individue trinitatis. Maximilianus eiusdem favente clementia etc. Es musste deshalb Lunson mit Benützung dieses Konzeptes (GG Bl. 15) einen kanzleigemässen Entwurf herstellen ebda Bl. 108.

6) Neues Archir 25. S. 347 ff. 
das in der Kanzlei K. Arnolfs ausgeführte Original war. Hier hatte also ganz so, wie es zur Zeit Maximilians I. die Regel war, die Partei (in diesem Falle der Abt von St. Gallen) der königlichen Kanzlei den Entwurf einer Privilegienbestätigung vorgelegt, der mit einigen stilistischen und sachlichen Abänderungen im Originaldiplom seine Ausfertigung und Vollziehung fand.

Solche Fälle lassen sich auch in anderen Kanzleien konstatiren. Tangl selbst verweist auf ein Beispiel aus der Zeit Otto's III., wo ein vom Erzbischof von Bremen eingereichtes Konzept als Vorlage für die Originalausfertigung henützt wurde (DO. III. 24).

Es würde hier zu weit führen und streng genommen in den Rahmen unserer Ausführungen nicht gehören, die Frage über die Anteilnahme der Empfänger an dem Urkundungsgeschäfte überbaupt zu erörtern. Sie spielt in der neueren Literatur über Diplomatik eine so grosse Rolle, dass es in diesem Zusammenhange wohl genügen dürfte, auf sie nur kurz hinzuweisen. War es also verwunderlich, dass, wenn man die Urkunden selbst konzipirte, man auch die Konzepte in der Kanzlei einreichte? Man sandte ja auch die Abschriften der zu bestätigenden Urkunden ein. Solche Kopien finden sich in den zu Pisa aufbewahrten Resten des deutschen Reichsarchivs Heinrichs VII. Sie sind dort sogar mit Anweisungen für die Kanzleibeamten versehen ${ }^{1}$ ). Dasselbe wiederholt sich dann in den Kanzleien Karls IV. und seiner Nachfolger ${ }^{2}$ ).

Aber auch, dass die Parteien selbstgefertigte Konzepte einsenden, ist nichts mehr seltenes und lässt sich aus der Zeit Sigismunds des öftern nachweisen ${ }^{3}$ ). Es ist ja begreiflich, dass mit der Zunahme des Urkundenwesens, wenigstens seinem äusserlichen Umfange nach, und den Anwachsen des Schreibgeschäftes diese Übung von den Kanzleibeamten als eine erwünschte Entlastung ihrer Tätigkeit daukbar empfunden wurde.

Interessant ist es übrigens, dass sich diese Gepflogenheit auch in der päpstlichen Kanzlei und zwar bereits im 12. Jahrhundert nachweisen lässt4). Aber sowohl bei diesem von Kehr entdeckten Konzepten, wie auch bei dem Entwurfe, den Tangl beschreibt, war es das Archir des Empfängers, in dem diese Stücke aufbewahrt wurden.

1) Sitzungsber. d. Wiener Akad. 14, 158.

2) Lindner, Das Urkundenwesen Karls IV. und seiner Nachfolger S. 148 f.

9) Ebda S. 150. Vgl. auch Janssen Frankfurts Reichskorrespondenz I. S. 360 f. Nr. 667 und 668, wo zwei Entwürfe von Privilegien für Frankfurt abgedruckt sind, die der Rat der Stadt an den Protonotar Kaspar Schlick sandte.

4) Kehr, Die Minuten von Passignano, Quellen und Forschungen aus ital. Archiven und Bibl. VII. S. 8 ff. 
Daraus schliesst Tangl, dass die Partei durch die Entrichtung der Konzepttaxe sich ein gewisses Eigentumsrecht an dem Konzepte erworben habe und die Prokuratoren die schliesslichen Empfänger der Entwürfe waren. Dies mag ja für jene frühen Zeiten Geltung haben, in der Reichskanzlei Maximilians I. war dies sicher nicht der Fall und widerspräche auch der in der Kanzleiordnung niedergelegten Bestimmung, dass selbst jene Minuten, die nicht eigens registrirt zu werden brauchen, verwahrt werden sollen. 